\title{
Experiencias
}

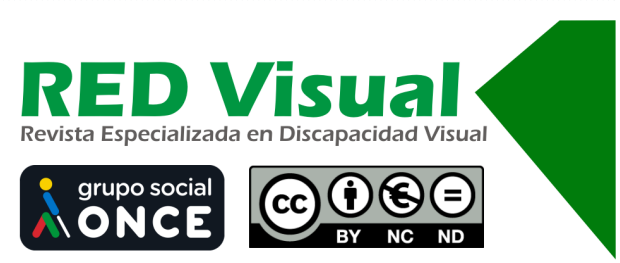

Recepción: 09-12-2020 Aceptación: 06-05-2021

\section{Experiencia piloto para la difusión del servicio de voluntariado ${ }^{1}$}

\section{Pilot experience for publicising the volunteer service}

\author{
M.C. Domínguez Olaya
}

\section{Resumen}

Este trabajo consiste en una campaña de difusión del servicio de voluntariado que se ha realizado en las zonas rurales más alejadas de la capital de la provincia de Huelva, con la colaboración de las distintas entidades públicas y privadas de la zona y con el objetivo de acercar los servicios de la ONCE a los afiliados que allí residen. Se inicia con la entrada en vigor de la nueva normativa reguladora de la actuación de la ONCE como entidad de voluntariado (circular n. ${ }^{0}$ 1/2020 de 13 de enero de la Dirección General Adjunta de Servicios Sociales para Afiliados de la ONCE), la cual introduce como novedad, entre otras cuestiones, la creación del programa de «Difusión». Los resultados de esta práctica, como se explicará más adelante, han ido mucho más allá de la difusión del servicio de voluntariado, cuyo primer objetivo es captar voluntarios y beneficiarios en la zona para poner en marcha el servicio.

\section{Palabras clave}

Voluntariado. Difusión. Ámbito rural. Coordinación.

\section{Abstract}

This work consists of a campaign to publicise the volunteer service which has been carried out in the rural areas farthest from the capital city of the province of Huelva, with the collaboration

1 Trabajo galardonado con un accésit en la categoría General del v Concurso de Experiencias de Innovación y Buenas Prácticas en Servicios Sociales de la ONCE, presentado bajo el mismo título. 
of public and private organisations in the area and with the aim of bringing ONCE services closer to the members who live there. It began with the entry into force of the new regulations governing the ONCE'S activities as a volunteer organisation (circular no. 1/2020 of 13 January from the ONCE'S Deputy Director General for Social Services for Members), which introduced, among other matters, the creation of the «Difusión» programme as a new feature. The results of this practice, as will be explained later, have gone far beyond the publicising of the volunteer service, the first objective of which is to recruit volunteers and beneficiaries in the area to set up the service.

\section{Key words}

Volunteers. Publicity. Rural areas. Coordination.

\section{Presentación y justificación}

La experiencia que aquí se presenta nace de la necesidad expresada por determinados afiliados residentes en la sierra de Huelva de poder acceder a los recursos de la ONCE; más concretamente, al servicio de voluntariado.

Partimos de que en la provincia de Huelva hay una población muy dispersa de afiliados, y tan solo una agencia de la ONCE, en la Palma del Condado, que pertenece a la Delegación Territorial de Andalucía, sita en Sevilla, aunque esta agencia abarca afiliados residentes en la provincia de Huelva.

En los municipios de la sierra, las comunicaciones con la capital onubense, donde tiene su sede la Dirección de Apoyo de la ONCE (DAP), son muy limitadas.

No disponer de un adecuado sistema público de transporte que responda a las necesidades de las personas que viven en esta zona, junto a un modelo de movilidad basado en el uso del vehículo privado, excluye a muchas personas, especialmente a nuestros afiliados, por lo que la autonomía en movilidad se convierte en una condición necesaria para la calidad de vida en los espacios rurales de nuestra provincia, en la medida en que de ella depende el acceso a los servicios.

Como consecuencia de la deficiente red de transporte público, los afiliados que residen allí tienen un difícil acceso a los servicios en general (sociales, sanitarios, 
culturares, deportivos, etc.) y, particularmente, a los servicios que presta la ONCE; más concretamente, al servicio de voluntariado.

En esta zona contamos con un total de 57 afiliados adultos distribuidos en 22 localidades distintas.

Iniciamos esta experiencia en los municipios de Aracena y Cortegana, dos de las localidades con mayor número de habitantes y afiliados a la ONCE, ya que, en el caso de Aracena, es cabeza de partido de 31 municipios, ejerciendo de capital administrativa, comercial y turística de toda la comarca serrana.

Partiendo de esta realidad, y realizando un trabajo de equipo, la trabajadora social, el instructor tiflotécnico y braille y la técnica en rehabilitación integral que llevan la zona, analizan esta población, comprobando que existen necesidades que podrían ser cubiertas a través del servicio de voluntariado de la ONCE y que no están siendo cubiertas porque no se cuenta con ningún voluntario en la zona, por lo que la primera tarea a realizar es conocer y valorar qué entidades públicas y privadas prestadoras de servicios sociales existen en la zona y contar con ellos para realizar una campaña de difusión del servicio de voluntariado de la ONCE.

\section{Objetivos}

Esta campaña se realiza con un doble objetivo, captar voluntarios y captar afiliados potencialmente beneficiarios del servicio de voluntariado de la ONCE, objetivos que se concretan en:

- Difundir el servicio de voluntariado de la ONCE, llegando al mayor número de personas.

- Captar voluntariado en la zona.

- Captar afiliados potencialmente beneficiarios del servicio de voluntariado.

- Lograr que los afiliados de la zona se conozcan.

- Transmitir a la población en general, y a los afiliados en particular, una imagen de la ONCE como entidad que se coordina y comparte objetivos con instituciones 
públicas o privadas (ayuntamiento, centros de servicios sociales de la diputación provincial, asociaciones, etc.) para el logro del bienestar de sus afiliados.

- Acercar a nuestros afiliados los servicios sociales de la ONCE.

\section{Población destinataria de la experiencia}

La población destinataria de esta práctica son todos los afiliados mayores de 18 años de la zona de la sierra de Huelva, cualquier persona residente en la misma, ayuntamientos, centros de servicios sociales comunitarios, centros de salud y cualquier otra entidad que, de alguna manera, desconozca a la ONCE como entidad prestadora de servicios sociales.

\section{Temporalización y fechas de realización}

Esta práctica se inicia con la entrada en vigor de la circular n. ${ }^{0} 1 / 2020$ de 13 de enero de la Dirección General Adjunta de Servicios Sociales para Afiliados, la cual introduce como novedad la creación del programa de «Difusión». ${ }^{2}$

La actividad que se describe en estas páginas se realiza de forma más intensa durante el estado de alarma provocado por la crisis sanitaria, ya que, durante esos días, en los contactos que se realizan con los afiliados desde distintas instancias de la ONCE, como, por ejemplo, el Plan ONCERCA, ${ }^{3}$ los distintos técnicos del equipo y de la Sección de Unidad Progresista de Pensionistas de la ONCE (SUPO), ${ }^{4}$ entre otros, se pone de manifiesto la relevancia del servicio de voluntariado.

No se puede fijar una fecha para finalizar esta actividad, ya que debe ser generalizada, aplicable en cualquier zona y continuada en el tiempo.

2 Su finalidad es difundir el voluntariado entre las personas afiliadas a la ONCE y sus familias para garantizar que conozcan este servicio y puedan acceder a él. Igualmente, dentro de sus objetivos se encuentra la difusión del servicio para captación de nuevos voluntarios.

3 EI Plan ONCERCA consiste en una serie de contactos telefónicos y/o presenciales con las personas afiliadas a la ONCE con el objetivo de alcanzar una relación constante y de calidad con todas ellas, transmitiendo sentido de pertenencia a la misma y recogiendo y canalizando necesidades, inquietudes, sugerencias e impresiones de las personas afiliadas, contribuyendo así al mantenimiento de la actualización de su censo, además de incrementar los niveles de conocimiento de la cultura institucional.

4 SUPO es el cauce de representación y participación de las personas mayores en la ONCE, establecido principalmente para la defensa de los intereses de este colectivo.

Domínguez, M. C. (2021). Experiencia piloto para la difusión del servicio de voluntariado. RED Visual: Revista Especializada en Discapacidad Visual, 78, 102-110. https://doi.org/10.53094/BSQZ5076. 


\section{Metodología}

Cuando se inicia este trabajo de difusión, a raíz de la publicación de la nueva normativa de la ONCE como entidad de voluntariado, se planificaron una serie de actividades en la zona de la sierra, tales como charlas informativas en ciclos formativos de atención a la dependencia, escuelas de adultos, centros de participación activa, hogares de pensionistas, asociaciones, hermandades de Semana Santa, Cáritas, etc. (todo ello contando con la colaboración de algún voluntario que pudiera transmitir su experiencia a los potenciales voluntarios).

También se planificó una reunión informativa con los técnicos del centro de servicios sociales de la sierra, la trabajadora social de los centros de salud, los ayuntamientos, etc., ya que, en otras ocasiones, se ha colaborado con ellos a la hora de organizar actividades en esa zona con motivo del Día Internacional de las Personas con Discapacidad.

Semanas antes de que se decretase el estado de alarma como consecuencia de la crisis sanitaria provocada por la covid-19, las posibilidades de realizar actividades grupales presenciales se fueron reduciendo, quedando finalmente anuladas por la situación que se estaba viviendo.

Figura 1. Cartel de Voluntariado ONCE

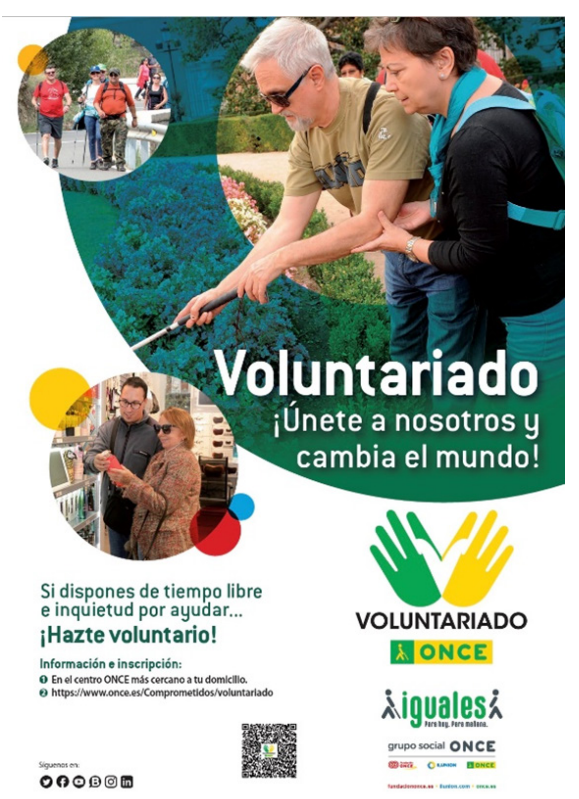

Domínguez, M. C. (2021). Experiencia piloto para la difusión del servicio de voluntariado. RED Visual: Revista Especializada en Discapacidad Visual, 78, 102-110. https://doi.org/10.53094/BSQZ5076. 
Llegó el confinamiento y, como consecuencia, el teletrabajo, por lo que la metodología prevista para difundir el servicio de voluntariado también debía adaptarse a las nuevas circunstancias.

Se utilizaron como herramientas fundamentalmente el teléfono, el correo electrónico y la mensajería instantánea para lograr los objetivos. Se envió por e-mail a los centros de servicios sociales, ayuntamientos y centros de salud, entre otros, el cartel con el que la ONCE difunde el servicio de voluntariado (Figura 1).

Se contactó telefónicamente con las trabajadoras sociales de ayuntamientos y centros de salud con mayor población para informarles de las necesidades de nuestros afiliados residentes en su localidad y de la carencia de voluntarios en la zona.

La respuesta de los ayuntamientos fue muy positiva, fundamentalmente la del de Aracena, que superó las expectativas, ya que, utilizando los documentos de difusión de la ONCE (cartel de voluntariado y folleto informativo de voluntariado en castellano), lo anunciaron en sus redes sociales, televisión local y periódico digital de la provincia (Figura 2).

Figura 2. Anuncio del periódico digital Huelva24.com

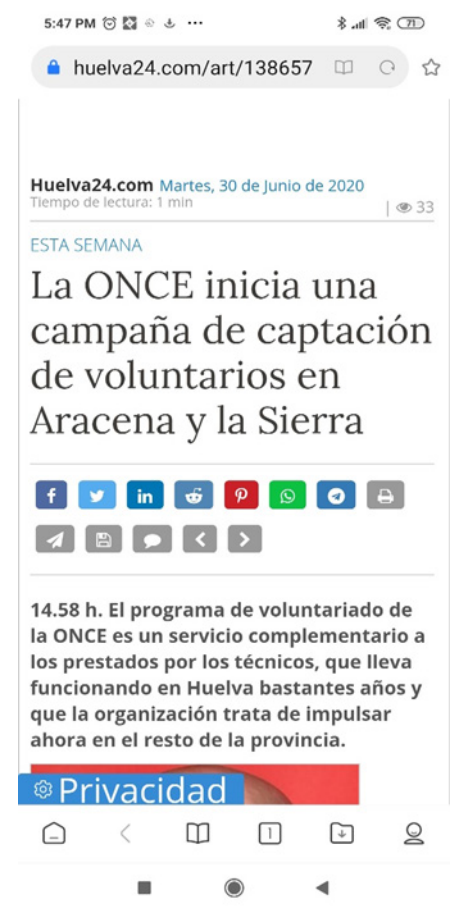


Del mismo modo, se contactó con las trabajadoras sociales del centro de salud y de los centros de servicios sociales para pedirles colaboración en la difusión de nuestro servicio de voluntariado. Se les facilitó el material de difusión y la respuesta fue también muy positiva.

Por otra parte, y dado que la ONCE tiene convenio con la Cruz Roja, se contactó telefónicamente con la asamblea de la Cruz Roja en las dos localidades en la que tiene sede, Aracena y Cortegana, con el objetivo de reactivar dicho convenio, ya que, en estas asambleas, lo desconocían. Acordamos una reunión presencial una vez que las circunstancias lo permitieran.

Una vez levantadas las restricciones que se impusieron durante el estado de alarma, se realizaron las siguientes actividades de forma presencial:

- Reunión en las dos sedes de la Cruz Roja de la zona para plantear las necesidades de nuestros afiliados y la colaboración real que la Cruz Roja podía ofrecer.

- Se realizó una actividad formativa por parte de la técnica de rehabilitación integral de la DAP para voluntarios de la Cruz Roja en la zona.

- Reunión con la psicóloga de la asociación Acaminar (trabaja con personas con discapacidad) a nivel local, que cuenta con un servicio de voluntariado y con socios afiliados a la ONCE. El objetivo de esta reunión es dar a conocer nuestros servicios y que nos ayudasen a captar voluntarios.

- Reunión con la trabajadora social del ayuntamiento de Aracena, localidad a la que nuestros afiliados acuden para la mayor parte de las gestiones que tienen que realizar y que están relacionadas con la administración pública (Seguridad Social, Agencia Tributaria, etc.). En esta reunión se plantean las dificultades con las que nos encontramos para poner en marcha el servicio de voluntariado al no contar la ONCE con una sede cercana (no disponemos de un espacio físico para realizar entrevistas a los voluntarios, impartir formación, realizar reuniones, etc.).

- Distribución del material de difusión de la ONCE entre distintos comercios, bares, centros deportivos, etc., de la zona. 
- Se ha realizado una reunión informativa con afiliados que viven en la misma zona y no se conocían. En esta reunión se presentó al voluntario que presta servicios en la zona y entre los afiliados formaron un grupo de WhatsApp para mantener el contacto. El ayuntamiento cedió un pabellón para poder realizar esta reunión con todas las condiciones necesarias para realizarla con total seguridad, reunión en la que también estuvo la trabajadora social municipal para recibirnos.

Se contactó telefónicamente con Enclave Deportivo, una empresa privada de la zona, que, a través del senderismo, entre otras cosas, da a conocer la naturaleza y el patrimonio de la provincia. El objetivo en este caso era llegar a los clientes de esta empresa para captar voluntariado entre ellos, y que, de este modo, los afiliados de la zona pudieran participar en este tipo de actividades con el apoyo de un voluntario.

También, a través de contacto telefónico y por mensajería instantánea, se ha informado del servicio de voluntariado a la sede de Cáritas en la zona y a una hermandad de Semana Santa, mostrándonos una actitud de colaboración en todo momento.

\section{Resultados}

La difusión del servicio no llega tan solo a la población potencialmente voluntaria, sino también a los afiliados potencialmente beneficiarios del servicio, ya que son también informados por técnicos de ayuntamiento, del centro de servicios sociales, etc., profesionales en los que confían nuestros afiliados. Por lo tanto, se ha llegado a un mayor número de personas de las que hubiésemos llegado sin contar con la colaboración de estas entidades.

Se ha recuperado la colaboración de la Asamblea de la Cruz Roja en la zona: treinta voluntarios de la Cruz Roja de las asambleas de Cortegana y Aracena han sido formados en pautas de comportamiento con personas con discapacidad visual y técnica guía, y ahora podemos llevar a la práctica el Convenio de Colaboración firmado entre la ONCE y Cruz Roja Española con mayor calidad en la atención.

También contamos con un voluntario de la ONCE en la zona y otro en proceso de formación. A través del voluntario, como nexo entre distintos afiliados de la zona, se han fomentado las relaciones entre estos, comenzado así a funcionar el servicio de 
voluntariado donde no existía, aumentando, a su vez, la demanda de los servicios de tiflotecnología y de ocio y tiempo libre.

Por otra parte, la empresa Enclave Deportivo, dedicada al senderismo entre otras actividades, ha realizado un vídeo promocional sobre su actividad en la zona, y en él hace un llamamiento a sus clientes para que colaboren como voluntarios de la ONCE.

Se puede destacar como aspecto muy positivo que la población de la zona, que, de forma mayoritaria, conocía a la ONCE casi exclusivamente a través de la venta de productos de juego, es consciente ahora de que la inversión que cada día realizan con la compra del cupón es devuelta a la sociedad en forma de servicios sociales.

\section{Conclusiones}

La metodología utilizada para realizar este trabajo de difusión se puede generalizar a todas las zonas rurales alejadas.

Contar con la colaboración de las entidades y profesionales valorados como «de confianza» por la población en general repercute positivamente en el sentimiento de cercanía a la ONCE de esta y de sus afiliados, como entidad prestadora de Servicios Sociales.

A corto plazo, los resultados de esta experiencia han sido más cualitativos que cuantitativos. Se ha sembrado una semilla cuyo fruto se verá reflejado no solo en la prestación de servicios a nuestros afiliados, sino también en la imagen del Grupo Social ONCE en la zona.

M. ${ }^{a}$ Carmen Domínguez Olaya. Trabajadora social y colaboradora del Servicio de Voluntariado. Dirección de Apoyo de la ONCE en Huelva. Calle Alameda Sundheim, 5; 21003 Huelva (España). Correo electrónico: mcdo@once.es. 